\title{
弛豫多铁性材料研究进展
}

\author{
魏永星 ${ }^{1}$, 靳长清 ${ }^{1}$, 曾一明 ${ }^{2}$ \\ (1. 西安工业大学 材料与化工学院, 西安 710021；2. 昆明贵金属研究所, 昆明 650106)
}

摘 要: 多铁性材料同时具有多种铁性(铁电性、铁磁性或铁弹性)的有序, 可实现电磁信号的相互控制, 成为近年来 研究热点。在具有成分无序的复杂体系中, 长程铁性有序有可能被打破, 材料将表现出驰豫特性。我们将至少存在 一种铁性弛豫特性的多铁性材料称之为驰豫多铁性材料。这类多铁性材料的极化强度(或磁化强度)在外加电场(或 外加磁场) 作用下响应更加灵敏, 其磁电耦合机制与长程有序的多铁性材料不同。本文结合国内外最新研究成果, 首先介绍了和弛豫铁性有序相关的物理概念，重点阐述了多铁性材料在铁电和铁磁双弛豫态下的磁电耦合机制; 然后，详细介绍了钻铁矿结构(包括 $\mathrm{PbB}_{1} \mathrm{~B}_{2} \mathrm{O}_{3}$ 基和 $\mathrm{BiFeO}_{3}$ 基材料)和非钻铁矿结构(包括层状 $\mathrm{Bi}$ 结构和非正常铁电 体)弛豫多铁性材料的研究进展; 最后，对该领域亟待解决的问题进行了展望。

关 键 词: 弛豫性; 多铁性材料; 磁电耦合; 纳米存储器件

中图分类号: TQ174 文献标识码: A

\section{Progress of Relaxor Multiferroic Materials}

\author{
WEI Yong-Xing ${ }^{1}$, JIN Chang-Qing ${ }^{1}$, ZENG Yi-Ming ${ }^{2}$ \\ (1. School of Materials and Chemical Engineering, Xi'an Technological University, Xi'an 710021, China; 2. Kunming Institute \\ of Precious Metals, Kunming 650106, China)
}

\begin{abstract}
The multiferroic material, which shows the coexistences of multi ferroic orders (ferroelectricity, ferromagnetism or ferroelasticity), can realize the mutual control of the electric and magnetic signals, and becomes one of the hottest research topics. The long-range ferroelectric or ferromagnetic order may be broken in compositionally disordered systems. In this situation, it is posssible that materials display relaxor behavior. Multiferroic materials possessiong at least one ferroic relaxor character can be named as relaxor multiferroics. The polarization (or magnetization) is very sensitive to the applied electric field (or magnetic field). Besides, the magnetoelectric coupling effect of relaxor multiferroics is different from that of multiferroics with long ferroic orders. In this paper, the most recent and important theoretical and experimental advances in this new research field are reviewed. Firstly, basic physical concepts of the relaxor ferroic orders and the different mechanism of the magnetoelectric coupling effect on materials are introduced with the coexistence of relaxor ferroelectric ordering and relaxor magnetic ordering. Then, the recent researches on two sorts of the relaxor multiferroics, including perovskite $\left(\mathrm{PbB}_{1} \mathrm{~B}_{2} \mathrm{O}_{3}\right.$ based and $\mathrm{BiFeO}$ based $)$ and non-perovskite (Bi-layered based and improperly ferroelectric based) structural materials, are reviewed. Finally, the further development of relaxor multiferroics is prospected.
\end{abstract}

Key words: relaxor behavior; multiferroics; magnetoelectric coupling effect; nano memory device

收稿日期：2016-11-25; 收到修改稿日期：2017-01-09

基金项目：国家自然科学基金青年项目(11404251, 51602242); 西安工业大学校长基金重点项目(XAGDXJJ15008) National Natural Science Foundation of China (11404251, 51602242); President's Fund of Xi'an Technological University (XAGDXJJ15008)

作者简介：魏永星(1985-), 男, 博士, 讲师. E-mail: weiyx1985@gmail.com 
多铁性材料是指同时存在两种或两种以上铁性 有序(铁电序、铁磁序、铁弹性)的材料, 可以实现电 信号和磁信号的交互控制, 在传感器、能量收集转 换器、可调微波器件和新型存储器等领域具有巨大 的应用前景 ${ }^{[1-4]}$ 。早在 1894 年, Curie 就预言了磁电 耦合效应的存在。从上个世纪末开始, 科研工作者 就致力于多铁性材料磁电耦合的研究, 但是未取得 突破性的进展。直到 2003 年, 美国 Ramensh 研究组 和日本 Kimura 研究组分别在钻钛矿结构的 $\mathrm{BiFeO}_{3}$ 薄膜和正交结构的 $\mathrm{TbMnO}_{3}$ 上发现了大的磁电耦 合效应, 从而掀起了多铁性材料的研究热潮 ${ }^{[5-6]}$ 。十 几年来, 多铁性材料的理论和实验研究已取得了 骄人的成绩。关于这一部分内容, 我国南京大学刘俊 明 ${ }^{[7-8]}$ 、清华大学南策文 ${ }^{[4]}$ 、中国科学院靳常青 ${ }^{[9]}$ 、华

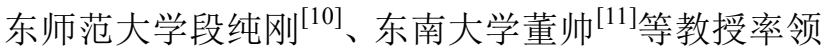
的研究组均有很好的总结 ${ }^{[4,7-11]}$ 。

本文要讨论的弛豫多铁性材料与常规意义的多 铁性材料有着明显的不同, 当材料的长程铁性有序 被打破而表现出纳米尺度的短程铁性有序时，材料 将表现出弛豫特性 ${ }^{[12]}$ 。我们将至少存在一种铁性弛 豫特性的多铁性材料称为弛豫多铁性材料。这里所 指的铁性弛豫特性, 既包括铁电弛豫性和铁磁弛豫 性, 也包括铁弹弛豫性, 但由于铁弹弛豫性的多铁 性研究较少, 本文暂不讨论该方面的内容。从狭义 上讲, 弛豫多铁性材料可分为铁电弛豫性和长程铁 磁序(铁磁、亚铁磁、反磁)共存的材料、铁电性和 铁磁弛豫性共存的材料、铁电弛豫性和铁磁弛豫性 共存的材料。从相组成来看, 弛豫多铁性材料还包 括单相弛豫多铁性材料和复合弛豫多铁性材料, 本 文涉及的是单相弛豫多铁性材料。弛豫铁电和弛豫 铁磁性的共存, 使得铁性有序参数在电场和磁场下 的响应更加敏感, 具有重要的科学意义和实用价 值。在这一领域, 英国、德国、日本、中国和斯洛 文尼亚的科研工作者均做出了重要的贡献。本文将 主要对弛豫多铁性材料基本理论及发展现状进行介 绍, 并指出该领域可能的发展方向, 为相关研究工 作者提供参考。

\section{1 弛豫多铁性基本理论}

\section{1 弛豫铁电性和弛豫铁磁性}

弛豫铁电性一般出现在复杂化合物和固溶体中, 在同一晶体学位置上, 不同类型离子的共存导致原 子排列的无序性及化合价和键合 (共价键、离子键) 的混合, 从而引起化学和结构的不均匀性。在介电
行为上, 弛豫铁电体表现出宽化的弥散介电相变异 常峰, 介电常数峰值可以很大 $(>10,000)$, 介电常数 峰值对应的温度与频率相关 ${ }^{[13]}$ (图 1)。值得注意的 是, 弥散介电相变并不对应结构的相变。关于弛豫 铁电体的介电相变存在几种解释, 包括微畴-宏畴 转变理论 ${ }^{[14]}$, 超顺电态理论 ${ }^{[15]}$, 结构起伏理论以及 有序-无序转变模型 ${ }^{[16]}$ 。近年来, 科学界倾向于认为 弛豫铁电体的行为与极性纳米微区 (PNR) 相关 ${ }^{[13]}$ 。 当弛豫铁电体处于热遍历态时, 在微区内可能存在 扭曲的铁电结构, 但在宏观上表现为平均立方相, $P-E$ 曲线为无滞后的非线性关系, 剩余极化强度为 零, 压电系数为零。而随着温度降低, PNR 可以被冷 冻或者转变为铁电畴, 与之对应, 弛豫铁电体由弛 豫态转变到非热遍历态或者铁电态。弛豫铁电体介 电常数最大值的温度-频率关系可以通过 VogelFulcher(V-F)方程描述:

$$
f=f_{0} \exp \left[-E_{\mathrm{a}} / k_{\mathrm{B}}\left(T_{\mathrm{m}}-T_{\mathrm{f}}\right)\right],
$$

其中, $f$ 代表测量频率, $T_{\mathrm{m}}$ 代表介电异常峰峰值对应 的特征温度, $k_{\mathrm{B}}$ 为玻尔兹曼常数, $f_{0} 、 E_{\mathrm{a}}$ 和 $T_{\mathrm{f}}$ 为拟合 参数, $E_{\mathrm{a}}$ 为激活能, $T_{\mathrm{f}}$ 为冷冻温度, 代表由弛豫态转 变到非热遍历态的温度 ${ }^{[17]}$ 。非热遍历态下的 PNR 在电场作用下可以转变为铁电畴，因此，弛豫铁电 体在非热遍历态下表现出与正常铁电体相类似的电 学性能, 例如, 方形电滞回线和大压电常数等。

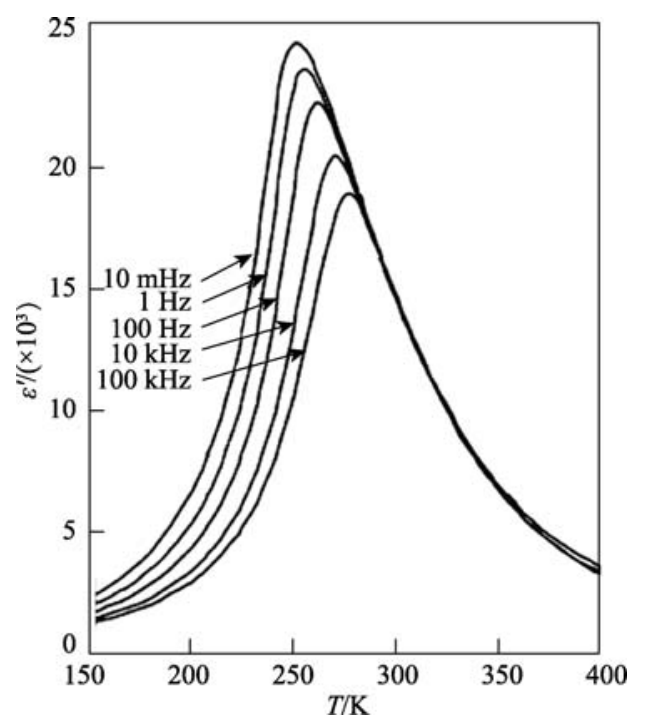

图 1 典型弛豫铁电体 $\mathrm{Pb}\left(\mathrm{Nb}_{2 / 3} \mathrm{Mg}_{1 / 3}\right) \mathrm{O}_{3}$ 不同频率下介电常 数与温度关系 ${ }^{[13]}$

Fig. 1 Temperature dependence of dielectric constant for classical relaxor ferroelectrics $\mathrm{Pb}\left(\mathrm{Nb}_{2 / 3} \mathrm{Mg}_{1 / 3}\right) \mathrm{O}_{3}$ at various frequencies $^{[13]}$

The classical relaxor ferroelectrics display the broad, frequency-dependent dielectric anomalies. The value of the maximum dielectric constant $\varepsilon_{\mathrm{m}}$ could reach above 10,000 . The relation between the $T_{\mathrm{m}}$ and frequency could be described by $V-F$ function 
类似地，还存在纳米尺度的短程磁有序 (NMR) 材料, 其磁性介于长程磁有序材料(铁磁体、亚铁磁 体和反铁磁体)和顺磁材料之间。科学家把短程磁有 序为主的材料称为弛豫铁磁体 ${ }^{[18-19]}$ 。通常采用自旋 玻璃态解释弛豫铁磁体的磁性。在弛豫铁磁体中, 电子的自旋不再统一排列, 而是随机取向, 这种随 机取向不再随时间的改变而变化, 即存在空间坐标 上的 “无序” 和时间坐标上的 “有序” (图 2)。与之 相应，材料表现出一些新奇的物理效应。比如，其交 流磁化率与温度曲线在玻璃态转变温度 $T_{\mathrm{g}}$ 附近表 现出异常峰, 并且该异常峰与频率明显相关, 可以 通过 V-F 方程来描述; 在很低的外加直流磁场作用 下，该异常峰就可被抹平。再比如，在带场冷却 $\mathrm{FC}$ 和零场冷却 ZFC 两种测量条件下, 磁化率在 $T_{\mathrm{g}}$ 以下 表现出明显的背离。

\section{2 短程电磁有序的耦合}

弛豫多铁性材料的磁电耦合机制是极其复杂 的。在这里, 我们仅讨论材料在铁电弛豫态(热遍历 态)和铁磁弛豫态(自旋玻璃态)共存下的磁电耦合。 铁电铁磁双弛豫态下，电致应变与 PNR-PNR 的相 互作用相关, 磁致应变与 MNR-MNR 的相互作用相 关，均为二次方效应，不存在时空反演对称的破缺。 宏观自发极化强度和自发磁化强度的缺失禁止线性 磁电耦合效应的出现。在这种情况下, 磁电耦合效 应与电致伸缩系数和磁致伸缩系数相关, 为间接的

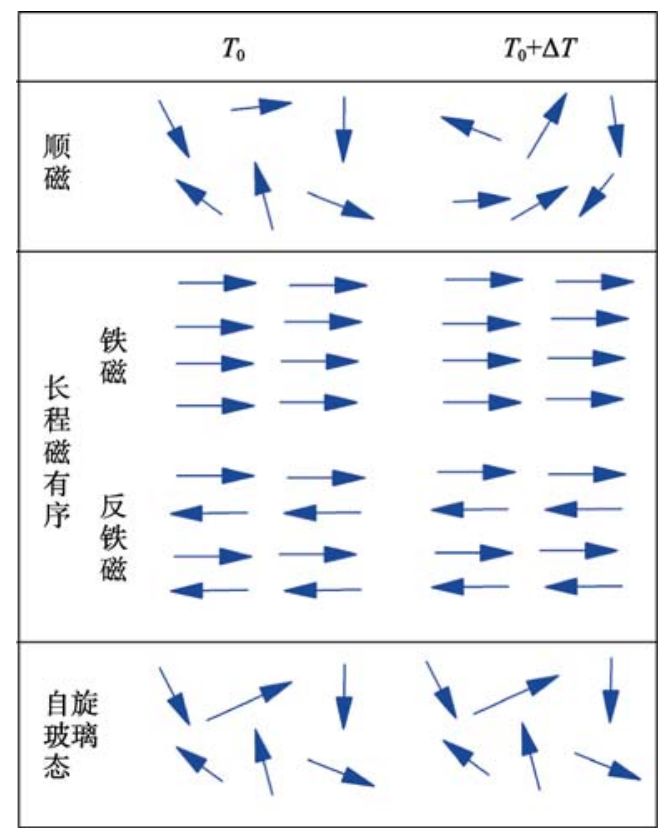

图 2 顺磁态、长程磁有序态(铁磁、亚铁磁和反铁磁)及自 旋玻璃态示意图

Fig. 2 Schematic representation of paramagnetic state, longranged magnetic state (ferromagnetic, ferrimagnetic, antiferromagnetic) and spin glass state
四次方效应 ${ }^{[12]}$ 。自由能表示如下:

$$
\begin{gathered}
F_{0}(P, M)=-E_{i} P_{i}-H_{j} M_{j}+\frac{1}{2}\left(\chi_{e}^{-1}\right)_{i j} P_{i} P_{j}+\frac{\mu_{0}}{2} \\
\left(\chi_{m}^{-1}\right)_{i j} M_{i} M_{j}+\frac{1}{4} b_{e} P^{4}+\frac{1}{4} b_{m} M^{4}
\end{gathered}
$$

其中, $\left(\chi_{e}^{-1}\right)_{i j}$ 代表 PNR 和 PNR 相互作用项， $\left(\chi_{\mathrm{m}}^{-1}\right)_{i j}$ 代表 MNR 和 MNR 的相互作用项，极化强度 $P$ 和磁 化强度 $M$ 的四次方的添加是为了确保系统的热力 学稳定性。与长程磁电序共存的自由能表达式不同, 上式不包含直接磁电耦合效应项 $\alpha_{i j} E_{i} H_{j}$ 。

\section{2 弛豫多铁性材料及磁电性能研究}

\section{1 钙钛矿结构型}

已报道的钙钛矿结构型弛豫多铁性材料基本可 以分为两类: 一类是 $\mathrm{PbB}_{1} \mathrm{~B}_{2} \mathrm{O}_{3}$ 基弛豫多铁性材料 (表 1), 另一类是 $\mathrm{BiFeO}_{3}$ 基弛豫多铁性材料(表 2)。 对于 $\mathrm{PbB}_{1} \mathrm{~B}_{2} \mathrm{O}_{3}$ 基钙钛矿材料, $\mathrm{B}_{1}$ 为铁磁活性离子, 如 $\mathrm{Fe}^{3+}, \mathrm{Co}^{3+}, \mathrm{Ni}^{3+}$ 等; $\mathrm{B}_{2}$ 通常为铁电或非铁电活性 离子, 铁电活性离子主要包括 $\mathrm{Ti}^{4+}, \mathrm{Nb}^{5+}, \mathrm{Ta}^{5+}$ 和 $\mathrm{W}^{6+}$ 。B 位不同性质离子的混合, 是其产生弛豫铁电 磁性的基础。而 $\mathrm{BiFeO}_{3}$ 基钙钛矿材料指的是 $\mathrm{BiFeO}_{3}$ 与其他钙钛矿材料形成的固溶体, 在 $\mathrm{A}$ 晶格位和 $\mathrm{B}$ 晶格位都可能存在两种或以上离子的混合。

\subsection{1 $\mathrm{PbB}_{1} \mathrm{~B}_{2} \mathrm{O}_{3}$ 基弛豫多铁性材料}

$\mathrm{B}$ 晶格位离子的属性和比例对 $\mathrm{PbB}_{1} \mathrm{~B}_{2} \mathrm{O}_{3}$ 型多 铁性材料有着重要的影响。 $\mathrm{PbFe}_{2 / 3} \mathrm{~W}_{1 / 3} \mathrm{O}_{3}$ 的 $\mathrm{B}$ 晶格 位铁磁活性离子 $\mathrm{Fe}^{3+}$ 和铁电活性离子 $\mathrm{W}^{6+}$ 比例为 2 : 1, 表现出短程极化有序 (极性纳米微区冷冻温度 $T_{\mathrm{f}}<300 \mathrm{~K}$ ) 和长程反铁磁有序 (反铁磁奈尔温度 $T_{\mathrm{N}}>$ $300 \mathrm{~K})$ 的共存 ${ }^{[20-22]}$ 。而对于 $\mathrm{B}$ 晶格位铁磁活性离子 $\left(\mathrm{Fe}^{3+}\right)$ 与铁电活性离子比例为 $1: 1$ 的 $\mathrm{PbFe}_{1 / 2} \mathrm{Nb}_{1 / 2} \mathrm{O}_{3}$ 和 $\mathrm{PbFe}_{1 / 2} \mathrm{Ta}_{1 / 2} \mathrm{O}_{3}$, 铁电序基本以长程有序为主, 铁 电结构为四方 (空间群 $\mathrm{P} 4 \mathrm{~mm}$ ) 相, 后者居里点低于 室温 ${ }^{[23-27]}$ 。在低温下, 二者还存在从四方对称性到 单斜对称性的相转变。近几年, 科研工作者对这三 种材料进行了深入研究, 研究结果表明, 它们除了 存在反铁磁有序外，在极端低温下，还存在自旋玻 璃态 ${ }^{[28-31]}$ 。最近, $\mathrm{Ye}$ 研究组 ${ }^{[31]}$ 对 $\mathrm{PbFe}_{2 / 3} \mathrm{~W}_{1 / 3} \mathrm{O}_{3}$ 出现 的自旋玻璃态现象进行了解释, 如图 3 所示。由于 在 $\mathrm{PbFe}_{2 / 3} \mathrm{~W}_{1 / 3} \mathrm{O}_{3}$ 的 $\mathrm{B}$ 晶格位同时存在铁磁性 $\mathrm{Fe}^{3+}$ 离子和非铁磁性 $\mathrm{W}^{6+}$ 离子, $\mathrm{Fe}^{3+}$ 的自旋不可能是完全 的反铁磁有序。有一小部分 $\mathrm{Fe}^{3+}$ 自旋不能与邻近的 $\mathrm{Fe}^{3+}$ 自旋反平行排列, 称之为受挫的 $\mathrm{Fe}^{3+}$ 自旋。 $\mathrm{PbFe}_{2 / 3} \mathrm{~W}_{1 / 3} \mathrm{O}_{3}$ 的自旋玻璃态与受挫的 $\mathrm{Fe}^{3+}$ 自旋相关。 


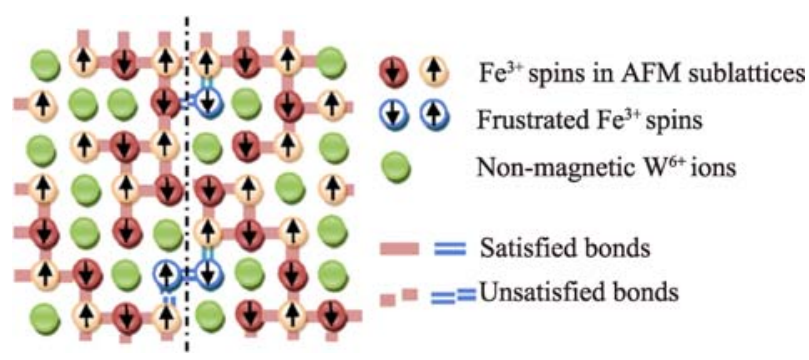

图 $3 \mathrm{PbFe}_{2 / 3} \mathrm{~W}_{1 / 3} \mathrm{O}_{3}$ 中 $\mathrm{Fe}^{3+}$ 自旋排列示意图, 在反铁磁子晶 格中存在挫败的 $\mathrm{Fe}^{3+}$ 的自旋 ${ }^{[31]}$

Fig. 3 Schematic representation of $\mathrm{Fe}^{3+}$ spins arrangement for $\mathrm{PbFe}_{2 / 3} \mathrm{~W}_{1 / 3} \mathrm{O}_{3}$, The frustrated $\mathrm{Fe}^{3+}$ spins appear in AFM sublattices ${ }^{[31]}$

2007 年, Levstik 研究组 ${ }^{[32]}$ 发现, 在 $\mathrm{PbFe}_{1 / 2} \mathrm{Nb}_{1 / 2} \mathrm{O}_{3}$ 中引入 $20 \mathrm{~mol} \%$ 的 $\mathrm{PbMg}_{1 / 2} \mathrm{~W}_{1 / 2} \mathrm{O}_{3}, \mathrm{PbFe}_{1 / 2} \mathrm{Nb}_{1 / 2} \mathrm{O}_{3}$ 固 有的长程铁电序和长程反铁磁序均被打破, 表现出铁电 铁磁双弛豫现象。在极性冷冻温度附近, 还存在磁导率 的异常, 这可能来自于纳米尺度的极性微区和磁性微区 的耦合。该研究工作的发表, 带动了弛豫多铁材料研究 的发展。此后, $\mathrm{Pb}\left(\mathrm{Zr}_{0.53} \mathrm{Ti}_{0.47}\right)_{0.60}\left(\mathrm{Fe}_{0.5} \mathrm{Ta}_{0.5}\right)_{0.4} \mathrm{O}_{3}$ 、

$\mathrm{Pb}\left(\mathrm{Fe}_{0.66} \mathrm{~W}_{0.33}\right)_{0.8} \mathrm{Ti}_{0.2} \mathrm{O}_{3}$ 和 $\mathrm{Pb}\left(\mathrm{Fe}_{0.66} \mathrm{~W}_{0.33}\right)_{0.2}\left(\mathrm{Zr}_{0.53} \mathrm{Ti}_{0.47}\right)_{0.8} \mathrm{O}_{3}$ 等固溶体系的弛豫多铁性陆续受到关注 ${ }^{[33-37]}$ 。特别是, Kumar 等 ${ }^{[35-36]}$ 在研究 $\mathrm{Pb}\left(\mathrm{Fe}_{0.66} \mathrm{~W}_{0.33}\right)_{0.2}\left(\mathrm{Zr}_{0.53} \mathrm{Ti}_{0.47}\right)_{0.8} \mathrm{O}_{3}$ 薄膜的磁电耦合效应时, 发现了一个有趣的现象: 该薄膜表现出弛豫铁电性, 在室温下 PNR 被冻结。 随着外加磁场强度的增强, 薄膜的剩余极化强度减 少, 当磁场强度施加到 $0.5 \mathrm{~T}$ 时, 薄膜的剩余极化强 度突降至 0 (图 4); 当撤去磁场时, 剩余极化强度 接近初始值。他们认为, 这种磁致效应与相变无关, 而是源于自旋和极化弛豫的强烈耦合。该工作表明, 在磁场和电场的协同作用下，弛豫多铁性材料可能 存在三种存储态 $\left(\mathrm{P}_{\mathrm{r}}\right.$ 态、 $-\mathrm{P}_{\mathrm{r}}$ 态和 0 态), 在新型的非 挥发存储器领域具有巨大的应用前景(图 5)。但是, 当该材料以块体陶瓷的形式存在时, 表现出长程有 序的铁电性能，上述效应消失 ${ }^{[38]}$ 。

\subsection{2 $\mathrm{BiFeO}_{3}$ 基弛豫多铁材料}

$\mathrm{BiFeO}_{3}$ 室温下具有长程有序的铁电三方结构, 空间群为 $\mathrm{R} 3 \mathrm{c}, \mathrm{Bi}^{3+}$ 沿着 $[111]_{\mathrm{c}}$ 方向偏离理想立方钙 钛矿中心, 理论自发极化强度为 $110 \mu \mathrm{C} / \mathrm{cm}^{2}$ 。无论 以单晶、薄膜还是块体的形式存在, $\mathrm{BiFeO}_{3}$ 均表现 出大的剩余极化强度 ${ }^{[5,39-43]}$ 。在 $830 \sim 850^{\circ} \mathrm{C}$, 铁电三 方相转变为顺电正交相 ${ }^{[44]}$ 。此外, 它还具有 $\mathrm{G}$ 型反 铁磁长程有序结构。通过在 $\mathrm{BiFeO}_{3}$ 中的 $\mathrm{A}$ 晶格位 或 $\mathrm{B}$ 晶格位引入其他元素, 可能破坏其长程有序铁 电或反铁磁结构, 获得弛豫多铁性材料。

2011 年, 苗君研究组 ${ }^{[45]}$ 通过外延法成功生长了 $\mathrm{Bi}\left(\mathrm{Fe}_{0.5} \mathrm{Mn}_{0.5}\right) \mathrm{O}_{3}$ 薄膜, 其极化序和磁化序均是短程
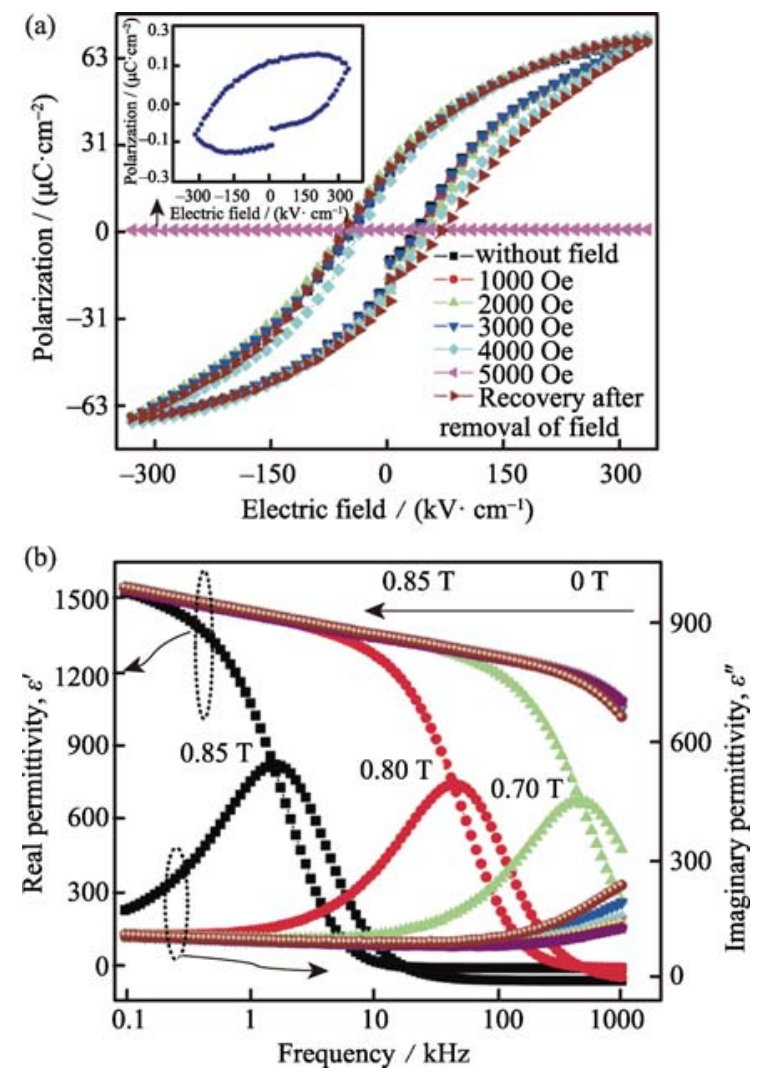

图 $4 \mathrm{~Pb}\left(\mathrm{Fe}_{0.66} \mathrm{~W}_{0.33}\right)_{0.2}\left(\mathrm{Zr}_{0.53} \mathrm{Ti}_{0.47}\right)_{0.8} \mathrm{O}_{3}$ 在磁场作用下的(a)极 化强度-电场曲线及(b)介电常数实部虚部与频率的关系 ${ }^{[35]}$

Fig. 4 (a) The $P$-E loops and (b) the real and imaginary part of dielectric constant as a function of frequencies with various magnetic fields for $\mathrm{Pb}\left(\mathrm{Fe}_{0.66} \mathrm{~W}_{0.33}\right)_{0.2}\left(\mathrm{Zr}_{0.53} \mathrm{Ti}_{0.47}\right)_{0.8} \mathrm{O}_{3}{ }^{[35]}$ Increase of the magnetic field $H$ leads to decrease in $P_{\mathrm{r}} . P_{\mathrm{r}}$ is nearly zero when $H$ reaches $0.5 \mathrm{~T}$. This effect disappears after magnetic field being removed. Correspondingly, the anomaly peak of the imaginary part for dielectric constant shifts to the low frequency side with $H$ increasing, which reveals the increase of the relaxation time

的，通过 Landau-Devonshire 理论，确定 PNR 的尺寸 为 $7 \sim 11 \mathrm{~nm}$ 。

在 $\mathrm{BiFeO}_{3}$ 中引入 $\mathrm{BaTiO}_{3}$, 可以形成 $\mathrm{BiFeO}_{3}$ $\mathrm{BaTiO}_{3}$ 固溶体系。研究发现，随着 $\mathrm{BiFeO}_{3}$ 含量增加， 该体系存在铁电一弛豫转变, 可以表现出弛豫铁电 态和亚铁磁性的共存 ${ }^{[46-49]}$ 。该体系三方-准立方相界 附近的 $0.67 \mathrm{BiFeO}_{3}-0.33 \mathrm{BaTiO}_{3}$ 以单晶形式存在时, 则表现出更加丰富的磁电性质，通过中子漫散射实 验，日本研究者证实了该单晶在 $600 \mathrm{~K}$ 附近存在 $8 \mathrm{~nm}$ 左右的 PNR(图 6) ${ }^{[50]}$ 。随着温度上升, PNR 变小, 在 $800 \mathrm{~K}$ 附近 PNR 消失。此外, 该单晶表现出超顺磁 性，而超顺磁性和纳米磁畴相关，尺寸也在 $8 \mathrm{~nm}$ 左 右。他们认为, 纳米磁畴是由 PNR 引起的, 也就是 说该单晶的超顺磁性的来源与 PNR 密切相关。

与 $\mathrm{BiFeO}_{3}-\mathrm{BaTiO}_{3}$ 不同, $\mathrm{BiFeO}_{3}-\mathrm{PbTiO}_{3}$ 体系存在 两种铁电相共存的准同型相界 MPB, 特别在该体系的 四方区域, 四方度 $c / a$ 随 $\mathrm{PbTiO}_{3}$ 含量减少而增加 ${ }^{[51-52]}$ 。 
表 $1 \quad \mathrm{PbB}_{1} \mathrm{~B}_{2} \mathrm{O}_{3}$ 基弛豫多铁材料磁电特性

Table 1 Polar and magnetic orderings of the $\mathrm{PbB}_{1} \mathrm{~B}_{2} \mathrm{O}_{3}$ based multiferroic materials

\begin{tabular}{|c|c|c|}
\hline Compositions & Polar ordering & Magnetic ordering \\
\hline $\begin{array}{l}\mathrm{PbFe}_{2 / 3} \mathrm{~W}_{1 / 3} \mathrm{O}_{3} \\
\text { crystals }^{[31]}\end{array}$ & $\begin{array}{l}\text { Ferroelectric relaxor } \\
T_{\mathrm{m}}=210 \mathrm{~K} @ 0.1 \mathrm{MHz} \\
T_{\mathrm{f}}=164 \mathrm{~K}\end{array}$ & $\begin{array}{l}\text { Anti-ferromagnetic } \\
T_{\mathrm{N}}=350 \mathrm{~K} \\
\text { Magnetic glass state } \\
T_{\mathrm{g}}=10 \mathrm{~K}\end{array}$ \\
\hline $\begin{array}{l}\mathrm{PbFe}_{0.5} \mathrm{Nb}_{0.5} \mathrm{O}_{3} \\
\text { ceramics }^{[23,29]}\end{array}$ & $\begin{array}{l}\text { Ferroelectric } \\
T_{\mathrm{m}}=373 \mathrm{~K}\end{array}$ & $\begin{array}{l}\text { Anti-ferromagnetic } \\
T_{\mathrm{N}}=153 \mathrm{~K} \\
\text { Magnetic glass state } \\
T_{\mathrm{g}}=10.6 \mathrm{~K}\end{array}$ \\
\hline $\begin{array}{l}\mathrm{PbFe}_{0.5} \mathrm{Ta}_{0.5} \mathrm{O}_{3} \\
\text { ceramics }{ }^{[27,30]}\end{array}$ & $\begin{array}{l}\text { Ferroelectric } \\
T_{\mathrm{m}}=259 \mathrm{~K}\end{array}$ & $\begin{array}{l}\text { Anti-ferromagnetic } \\
T_{\mathrm{N}}=153 \mathrm{~K} \\
\text { Magnetic glass state } \\
T_{\mathrm{g}}<10 \mathrm{~K}\end{array}$ \\
\hline $\begin{array}{l}0.8 \mathrm{PbFe}_{1 / 2} \mathrm{Nb}_{1 / 2} \mathrm{O}_{3} \\
-0.2 \mathrm{PbMg}_{1 / 2} \mathrm{~W}_{1 / 2} \\
\mathrm{O}_{3} \text { ceramics }^{[32]}\end{array}$ & $\begin{array}{l}\text { Ferroelectric relaxor } \\
T_{\mathrm{m}}=280 \mathrm{~K} @ 0.1 \mathrm{MHz} \\
T_{\mathrm{f}}=245 \mathrm{~K}\end{array}$ & $\begin{array}{l}\text { Magnetic glass state } \\
T_{\mathrm{g}}=25 \mathrm{~K}\end{array}$ \\
\hline $\begin{array}{l}\mathrm{Pb}\left(\mathrm{Fe}_{0.66} \mathrm{~W}_{0.33}\right)_{0.8} \\
\mathrm{Ti}_{0.2} \mathrm{O}_{3} \\
\text { thin films } \\
\end{array}$ & $\begin{array}{l}\text { Ferroelectric relaxor } \\
T_{\mathrm{m}}=350 \mathrm{~K} \\
@ 10 \mathrm{kHz} \\
T_{\mathrm{f}}=238 \mathrm{~K}\end{array}$ & Ferrimagnetic \\
\hline $\begin{array}{l}\mathrm{Pb}\left(\mathrm{Fe}_{0.66} \mathrm{~W}_{0.33}\right)_{0.2} \\
\left(\mathrm{Zr}_{0.53} \mathrm{Ti}_{0.47}\right)_{0.8} \mathrm{O}_{3} \\
\text { thin film }\end{array}$ & $\begin{array}{l}\text { Ferroelectric relaxor } \\
T_{\mathrm{m}}<600 \mathrm{~K} \\
\text { @ } 1 \mathrm{MHz}\end{array}$ & Ferrimagnetic \\
\hline $\begin{array}{l}\mathrm{Pb}\left(\mathrm{Zr}_{0.53} \mathrm{Ti}_{0.47}\right)_{0.60} \\
\left(\mathrm{Fe}_{0.5} \mathrm{Ta}_{0 \cdot 5}\right)_{0.4} \mathrm{O}_{3} \\
\text { thin films }{ }^{[37]}\end{array}$ & $\begin{array}{l}\text { Ferroelectric relaxor } \\
T_{\mathrm{m}}=390 \mathrm{~K} \\
@ 1 \mathrm{MHz} \\
T_{\mathrm{f}}=305 \mathrm{~K}\end{array}$ & Ferrimagnetic \\
\hline
\end{tabular}

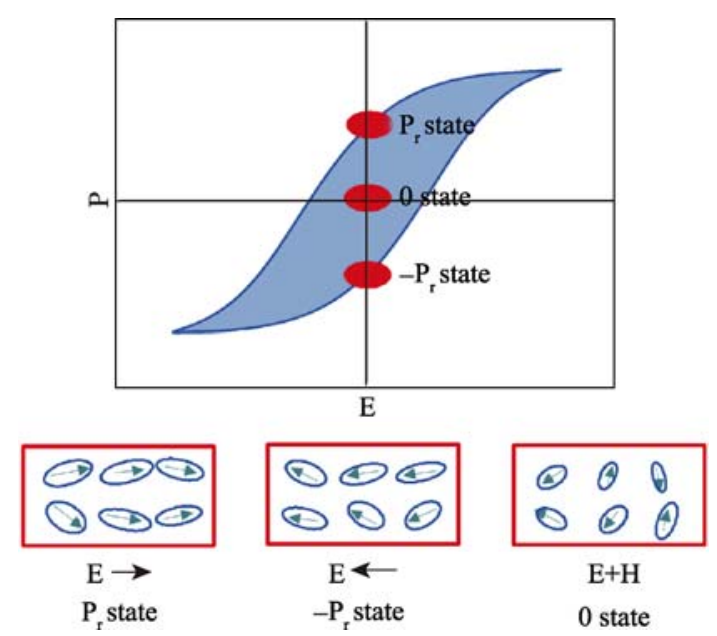

图 5 基于弛豫多铁性材料的多态存储器

Fig. 5 Relaxor multiferroics for multi-states memory device

该体系表现出长程有序的铁电结构, 由于大的晶格畸 变, 极化反转较困难, 矫顽场强很大 ${ }^{[53]}$ 。但是, 通过化 学改性有可能压制该体系的晶格畸变, 继而表现出铁 电一弛豫相变。例如, $0.5 \mathrm{Bi}\left(\mathrm{Fe}_{0.5} \mathrm{La}_{0.5}\right) \mathrm{O}_{3}-0.5 \mathrm{PbTiO}_{3}$ 陶 瓷就表现出铁电弛豫性和亚铁磁性的共存 ${ }^{[54]}$ 。

$\mathrm{BiFeO}_{3}-\mathrm{Bi}_{1 / 2} \mathrm{Na}_{1 / 2} \mathrm{TiO}_{3}$ 和 $\mathrm{BiFeO}_{3}-\mathrm{Bi}_{1 / 2} \mathrm{~K}_{1 / 2} \mathrm{TiO}_{3}$ 固 溶体系相似, 均在 $\mathrm{BiFeO}_{3}$ 含量减少到一定程度时表 现出铁电弛豫行为 ${ }^{[55-59]}$ 。近期, 德国科研工作者将 Co
表 $2 \mathrm{BiFeO}_{3}$ 基弛豫多铁材料磁电特性

Table 2 Polar and magnetic orderings of the $\mathrm{BiFeO}_{3}$ based multiferroic materials

\begin{tabular}{lll}
\hline \multicolumn{1}{c}{ Compositions } & \multicolumn{1}{c}{ Polar ordering } & Magnetic ordering \\
\hline $\mathrm{Bi}\left(\mathrm{Fe}_{0.5} \mathrm{Mn}_{0.5}\right) \mathrm{O}_{3}$ & Ferroelectric relaxor & Magnetic glass state \\
thin films & $T_{\mathrm{m}}=440 \mathrm{~K}$ & $T_{\mathrm{f} 2}=122 \mathrm{~K}$ \\
& $@ 1 \mathrm{MHz}$ & \\
& $T_{\mathrm{f}}=314 \mathrm{~K}$ &
\end{tabular}

0.65 $\mathrm{BiFeO}_{3^{-}} \quad$ Ferroelectric relaxor Ferrimagnetic

$0.35 \mathrm{BaTiO}_{3} \quad T_{\mathrm{m}}=687 \mathrm{~K}$

ceramics $^{[47]} \quad$ @ $1 \mathrm{MHz}$

0.67 $\mathrm{BiFeO}_{3^{-}} \quad$ Ferroelectric relaxor Magnetic glass state

$0.33 \mathrm{BaTiO}_{3} \quad T_{\mathrm{m}}=650 \mathrm{~K}$

single crystal ${ }^{[50]} @ 0.1 \mathrm{MHz}$

$0.5 \mathrm{Bi}\left(\mathrm{Fe}_{0.5} \mathrm{La}_{0.5}\right) \mathrm{O}_{3}-$ Ferroelectric relaxor Ferrimagnetic

$0.5 \mathrm{PbTiO}_{3} \quad T_{\mathrm{m}}=520 \mathrm{~K}$

ceramics $^{[34]} \quad$ @ $0.1 \mathrm{MHz}$

$0.6 \mathrm{BiFeO}_{3}{ }^{-} \quad$ Ferroelectric relaxor Ferrimagnetic

$\begin{array}{ll}0.4 \mathrm{Bi}_{1 / 2} \mathrm{~K}_{1 / 2} \mathrm{TiO}_{3} & T_{\mathrm{m}}=703 \mathrm{~K} @ 0.1 \mathrm{MHz} \\ \text { ceramics }^{[55-56]} & T_{\mathrm{N}}<500 \mathrm{~K}\end{array}$

$0.4 \mathrm{BiFe}_{0.9} \mathrm{Co}_{0.1} \mathrm{O}_{3^{-}}$Ferroelectric relaxor Ferrimagnetic

$0.6 \mathrm{Bi}_{1 / 2} \mathrm{~K}_{1 / 2} \mathrm{TiO}_{3} \quad T_{\mathrm{m}}=693 \mathrm{~K} \quad T_{\mathrm{N}}=670 \mathrm{~K}$

ceramics $^{[59]} @ 0.1 \mathrm{MHz}$

$\mathrm{T}_{\mathrm{f}}<573 \mathrm{~K}$

引入 $\mathrm{BiFeO}_{3}-\mathrm{Bi}_{1 / 2} \mathrm{~K}_{1 / 2} \mathrm{TiO}_{3}$ 的 $\mathrm{Fe}$ 晶格位，制备了 $0.4 \mathrm{BiFe}_{0.9} \mathrm{Co}_{0.1} \mathrm{O}_{3}-0.6 \mathrm{Bi}_{1 / 2} \mathrm{~K}_{1 / 2} \mathrm{TiO}_{3}$ 弛豫多铁性陶瓷 ${ }^{[60]}$ 。 在室温下，该陶瓷处于非热遍历态。结合压电力显 微镜 PFM 和磁力显微镜 MFM，首次观察到了极性 纳米有序和磁化序的共存区, 称之为多铁簇。最重 要的是, MFM 和 PFM 测试结果还证实多铁簇可以 分别在电场和磁场作用下反转(图 7)。特别需要指出 的是，该陶瓷的逆磁电耦合系数 $\alpha$ 为 $1.0 \times 10^{-5} \mathrm{~S} / \mathrm{m}$, 是 已知单相多铁材料中最高的。基于弛豫多铁性薄膜, 作者还设计了一种磁电耦合记忆器件，信息可以储 存在由电场控制的磁性单元里。

除此之外, 德国 Kleeman 研究组还报道了 $\mathrm{Sr}_{0.98} \mathrm{Mn}_{0.02} \mathrm{TiO}_{3}$ 陶瓷的弛豫多铁性能 ${ }^{[61]}$ 。 $\mathrm{SrTiO}_{3}$ 被 称为先兆铁电体 ${ }^{[62]}$, 其极化序被量子浮动所压制, 即使在 $0 \mathrm{~K}, \mathrm{SrTiO}_{3}$ 也保持在顺电态。随后的研究发 现, 引入微量 $\mathrm{Ca}$ 就有可能使 $\mathrm{SrTiO}_{3}$ 发生量子顺电 态到极化有序态的转变 ${ }^{[63]}$ 。葡萄牙研究者发现, 该 转变也可通过微量 $M n$ 改性得到 ${ }^{[64-65]}$ 。鉴于 $M n$ 的 铁磁活性, Kleeman 研究组 ${ }^{[61]}$ 展开了 $\mathrm{Sr}_{0.98} \mathrm{Mn}_{0.02} \mathrm{TiO}_{3}$ 的多铁性能的研究, 并发现该陶瓷弛豫铁电态和自 旋玻璃态的共存。

\section{2 非钙钛矿结构型}

和钙钛矿结构型材料相比, 非钙铁矿结构材料 的弛豫多铁性的研究相对较少, 主要集中在 Aurivillius 结构材料和非正常铁电体上。已报道的 Aurivillius 结构单相多铁材料的通式大多可以表示 为 $\mathrm{Bi}_{4} \mathrm{Ti}_{3} \mathrm{O}_{12} \cdot n \mathrm{BiMO}_{3},(\mathrm{M}=\mathrm{Fe} 、 \mathrm{Mn})^{[66-68]}$ 。其中, 
(a)

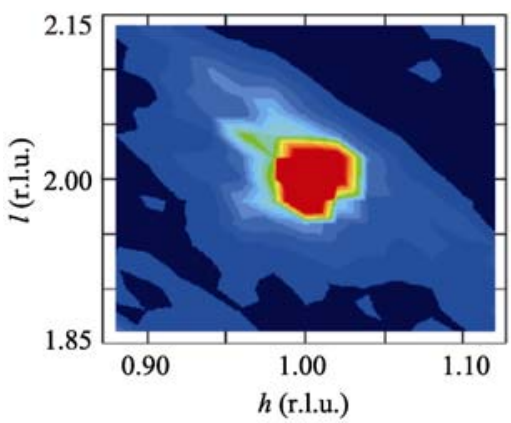

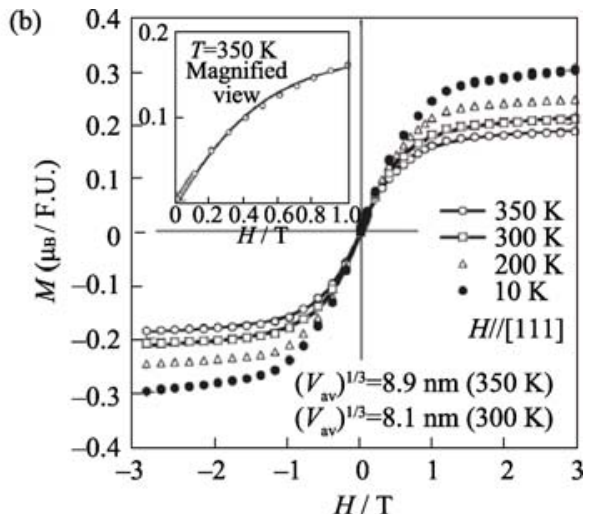

图 $60.67 \mathrm{BiFeO}_{3}-0.33 \mathrm{BaTiO}_{3}$ 单晶 $(\mathrm{a})(112)$ 衍射面在 $600 \mathrm{~K}$ 时的漫反射强度等高线和(b)在不同温度下的 $M-H$ 曲线 ${ }^{[50]}$

Fig. 6 (a) Contour plots of the diffuse intensities at $600 \mathrm{~K}$ around the (112) reflection and (b) M-H loops at various temperatures for the single crystal of $0.67 \mathrm{BiFeO}_{3}-0.33 \mathrm{BaTiO}_{3}{ }^{[50]}$ The crystal shows the strong nuclear diffuse scattering, with a correlation length of $8 \mathrm{~nm}$. It demonstrates the existence of the PNR. The M-H loops display the character of super-paramagnetism. The super-paramagnetism could be related to the short magnetic state. The fitting of Langevin function reveals the size of the short magnetic state is in the range of $8-9 \mathrm{~nm}$

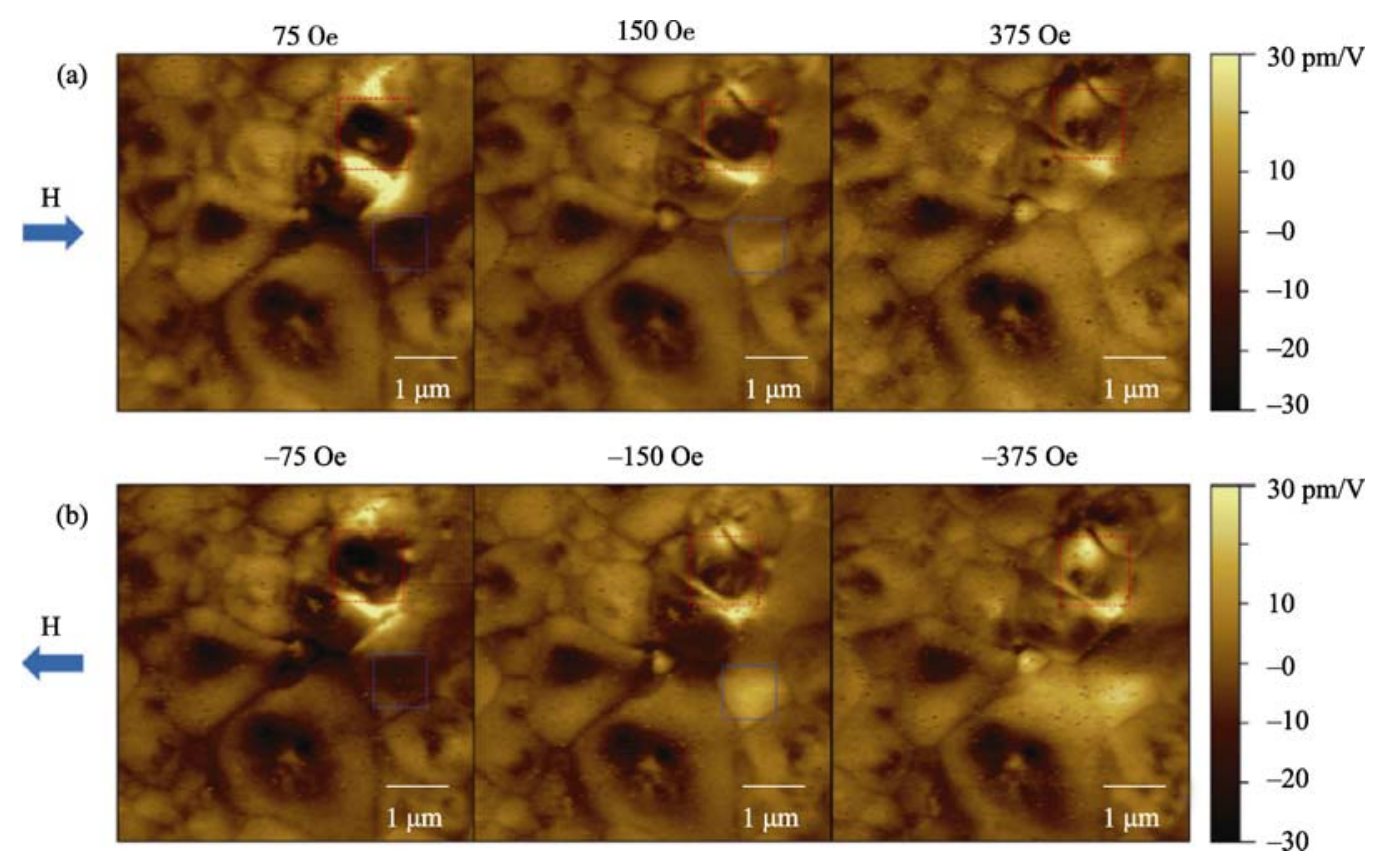

图 7 磁场作用下的同步压力电显微镜实验 ${ }^{[60]}$

Fig. 7 In situ PFM under magnetic field experiments ${ }^{[60]}$ The polarization switches obviously in the regions marked by blue and red rectangles

$\mathrm{Bi}_{5} \mathrm{Ti}_{3} \mathrm{FeO}_{15}\left(\mathrm{Bi}_{4} \mathrm{Ti}_{3} \mathrm{O}_{12} \cdot \mathrm{BiFeO}_{3}\right)$ 具有典型的 Aurivillius 结构, 其晶体结构如图 8(a)所示, 相邻两个 $\left[\mathrm{Bi}_{2} \mathrm{O}_{2}\right]^{2-}$ 层中的氧八面体层数为 4 。近期, 中国科学 技术大学研究人员对 $\mathrm{Bi}_{5} \mathrm{Ti}_{3} \mathrm{FeO}_{15}$ 的多铁性能进行 了系统的调查 ${ }^{[69]}$ 。扫描透射式电子显微镜 STEM 花 样证明 $\mathrm{Ti} / \mathrm{Fe}$ 沿[110]方向偏离理想位置中心，是该 材料铁电性的来源(图 8(b), (c))。能谱分析证实 $\mathrm{Bi}_{5} \mathrm{Ti}_{3} \mathrm{FeO}_{15}$ 中存在 $\mathrm{Fe}$ 的富集区域, 从而导致了短程 磁性有序。此外, 由于 $\mathrm{Bi}_{5} \mathrm{Ti}_{3} \mathrm{FeO}_{15}$ 的极化序和磁化 序都和同晶位离子相关, 使其极化强度和介电常数 对磁场非常敏感。
非正常铁电体的铁电性不是来源于铁电活性离 子与氧离子轨道杂化产生的共价作用, 而是与特定自 旋结构相关 ${ }^{[7]}$ 。2004 年, Nature 杂志报道了 Hemberger 研究组在铁磁材料 $\mathrm{CdCr}_{2} \mathrm{~S}_{4}$ 单晶方面的研究工作 ${ }^{[70]}$, 他们通过 $\mathrm{CdCr}_{2} \mathrm{~S}_{4}$ 的极化强度-电场关系和介电性 能-温度关系, 判断该材料存在弛豫铁电行为。Scott 研究组 ${ }^{[71]}$ 对该工作进行了质疑：该单晶的极化强 度-电场关系可能并不能确认其铁电行为, 而与有 较大损耗的介电材料类似。通过对介电数据的拟合, $\mathrm{CdCr}_{2} \mathrm{~S}_{4}$ 的冷冻温度低达 $-175 \mathrm{~K}$ (不可能存在)。 $\mathrm{CdCr}_{2} \mathrm{~S}_{4}$ 表现出的介电异常行为, 更像是来源于与 


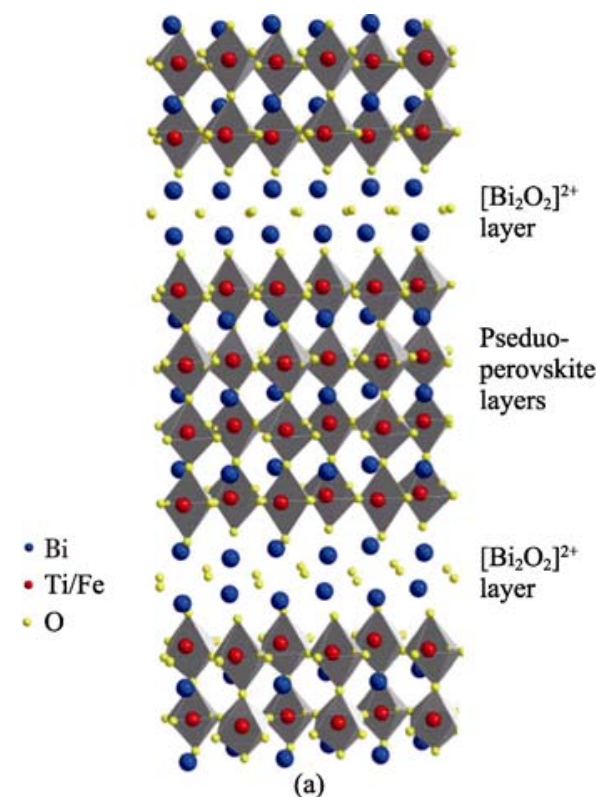

(a)

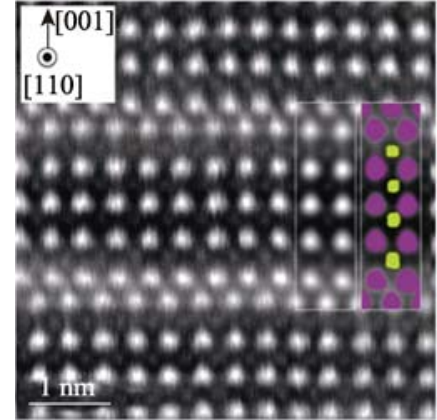

(b)

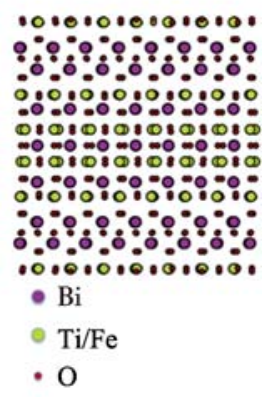

(c)
图 $8 \mathrm{Bi}_{5} \mathrm{Ti}_{3} \mathrm{FeO}_{15}$ (a)晶体结构(b)STEM 图谱和(c)原子占位 信息示意图 ${ }^{[68-69]}$

Fig. 8 (a) Crystal structure, (b) STEM pattern and (c) schematic illustration of atom position information of $\mathrm{Bi}_{5} \mathrm{Ti}_{3} \mathrm{FeO}_{15}{ }^{[68-69]}$ The crystal structure is obtained from ref [68]. Between the two $\left[\mathrm{Bi}_{2} \mathrm{O}_{2}\right]^{2-}$ layers, there are four $\mathrm{Ti}(\mathrm{Fe}) \mathrm{O}_{6}$ octahedra. The STEM measurement demonstrates $\mathrm{Ti} / \mathrm{Fe}$ atoms shift from ideal position along [110]

非均质半导体相关的 Maxwell-Wagner 效应。

$\mathrm{Fe}_{3} \mathrm{O}_{4}$ 是一种典型的磁性材料, 室温下具有反 式尖晶石 $\mathrm{AB}_{2} \mathrm{O}_{4}$ 结构, $\mathrm{A}$ 晶位由 $\mathrm{Fe}^{3+}$ 占据, $\mathrm{B}$ 晶位由 $\mathrm{Fe}^{3+}$ 和 $\mathrm{Fe}^{2+}$ 共同占据, 其磁性来源于 $\mathrm{Fe}^{2+}$ 和 $\mathrm{Fe}^{3+}$ 的交 换作用，居里点为 $860 \mathrm{~K}$ 。在 $120 \mathrm{~K}$ 左右, $\mathrm{Fe}_{3} \mathrm{O}_{4}$ 由反 式尖晶石相转变为电荷有序相, 其电荷有序与 $\mathrm{B}$ 晶 位 $\mathrm{Fe}^{2+}$ 和 $\mathrm{Fe}^{3+}$ 的排列相关 ${ }^{[72-73]}$ 。德国科研人员制备 了 $150 \mathrm{~nm}$ 厚度的 $\mathrm{Fe}_{3} \mathrm{O}_{4}$ 薄膜, 发现 $\mathrm{Fe}_{3} \mathrm{O}_{4}$ 在 $32 \sim 38 \mathrm{~K}$ 表现出 $4 \sim 8 \mu \mathrm{C} / \mathrm{cm}^{2}$ 的自发极化强度, 在介电常数与 温度的关系中表现出与频率相关的介电异常, 他们认 为与其本征的弛豫铁电行为相关 ${ }^{[74]}$ 。当施加平行于 薄膜方向的磁场后, $\mathrm{Fe}_{3} \mathrm{O}_{4}$ 的介电常数发生较大改变。

大多数非正常铁电体绝缘性差, 在确定其介电 行为时需要特别慎重。例如, 有研究者认为 $\mathrm{BiMn}_{2} \mathrm{O}_{5}$ 存在 PNR, 但给出的介电常数实部为 200 , 虚部却 高达 $100,000^{[75]}$ 。这里的介电行为已经不能反映本
征的介电弛豫，更有可能是 Maxwell-Wagner 效应的 影响。

\section{3 结束语}

关于 $\mathrm{Pb}\left(\mathrm{Fe}_{0.66} \mathrm{~W}_{0.33}\right)_{0.2}\left(\mathrm{Zr}_{0.53} \mathrm{Ti}_{0.47}\right)_{0.8} \mathrm{O}_{3}$ 薄膜和 $0.4 \mathrm{BiFe}_{0.9} \quad \mathrm{Co}_{0.1} \mathrm{O}_{3}-0.6 \mathrm{Bi}_{1 / 2} \mathrm{~K}_{1 / 2} \mathrm{TiO}_{3}$ 陶瓷的研究工作, 为驰豫型多铁性材料的发展指明了方向。但是, 必 须清醒地看到, 弛豫多铁性材料涉及到材料、物理、 计算机、电子信息等多学科领域的交叉, 研究尚处 于起步阶段，还存在不少亟待解决的问题。

1）弛豫多铁性研究的磁电效应包括长程铁电 有序和短程铁磁有序的耦合、短程铁电有序和长程 铁磁有序的耦合、短程电磁有序的耦合。特别对于 短程铁电有序, 还存在热遍历态和非热遍历态。这 些耦合效应，从宏观上看，涉及到不同的铁性序参 量的耦合; 从微观上看, 涉及到自旋、声子、电荷 或其它微观粒子或准粒子的相互作用, 不仅需要 在唯象理论上取得突破, 更需要在量子理论上继 续深入。

2）尽管已有不少弛豫多铁性材料的研究工作, 但是主要集中在其铁电性和磁性的弛豫行为研究上, 对于极化序和磁化序相互耦合的研究还比较欠缺, 在纳米尺度磁电耦合效应的实验研究则更乏陈可 举。对上述研究工作的推进将对弛豫多铁性材料的 应用产生巨大的促进作用。

3) 对于已发现的铁电铁磁短程有序共存的材 料，弛豫态和自旋玻璃态大多不在同一温度。开发 特定温度特别是室温下短程磁电弛豫态共存的弛豫 多铁材料并研究其 PNR 和 MNR 的耦合效应，具有 重要的科学意义。

4) $\left(\mathrm{Fe}_{0.66} \mathrm{~W}_{0.33}\right)_{0.2}\left(\mathrm{Zr}_{0.53} \mathrm{Ti}_{0.47}\right)_{0.8} \mathrm{O}_{3}$ 薄膜在室温下 发现的三存储态效应涉及非热遍历态下的 PNR 与 自旋的磁电耦合效应，该效应是否可以在其他具有 相似铁电磁特性的弛豫多铁性材料中重现, 还需要 进一步的研究。

5) 基于无铅环保性的考量, $\mathrm{BiFeO}_{3}$ 基无铅弛豫 多铁材料具有极大的研究价值。但是, 这一领域的 研究主要集中在陶瓷块体上, 而以薄膜和单晶形式 存在的弛豫多铁性材料的研究相对较少, 亟待展开。

\section{参考文献:}

[1] EERENSTEIN W, MATHUR N D, SCOTT J F. Multiferroic and magnetoelectric materials. Nature, 2006, 442(44): 759-765.

[2] SCOTT J F. Data storage. Multiferroic memories. Nature, 2007, 
6(4): 256-257.

[3] SPALDIN N A, FIEBIG M. The renaissance of magnetoelectric multiferroics. Science, 2005, 309(5733): 391-392.

[4] NAN C W. Research progress and future directions of multiferroic materials. Scientia Sinica Technologica, 2015, 45(4): 339-357.

[5] WANG J, NEATON J B, ZHENG H, et al. Epitaxial $\mathrm{BiFeO}_{3}$ multiferroic thin film heterostructures. Science, 2003, 299(5673): 1719-1722.

[6] KLMURA T, GOTO T, SHINTAL H, et al. Magnetic control of ferroelectric polarization. Nature, 2003, 426(6962): 55-58.

[7] LIU J M, NAN C W. Decade of multiferroic researches. Physics, 2014, 43(02): 88-98.

[8] WANG K F, LIU J M, WANG Y. Single phase multiferroic materialscoupling and adjusting between polar and magnetic order parameters. Chin. Sci. Bull, 2008, 53(10): 1098-1135.

[9] CHI Z H, JIN C Q. Recent advances in single-phase magnetoelectric multiferroics. Prog. Phys., 2007, 27(2): 225-238.

[10] DUAN C G. Progress in the study of magnetoelectric effect. Prog. Phys., 2009, 29(3): 215-238.

[11] DONG S, LIU J M. Multiferroic materials: past, present and future. Physics, 2010, 39(10): 714-715.

[12] PIRC R, BLINC R, SCOTT J F. Mesoscopic model of a system possessing both relaxor ferroelectric and relaxor ferromagnetic properties. Phys. Rev. B, 2009, 79(21): 214114.

[13] BOKOV A A, YE Z G. Recent progress in relaxor ferroelectrics with perovskite structure. J. Mater. Sci., 2006, 41(1): 31-52.

[14] YAO X, CHEN Z L, CROSS L E. Polarization and depolarization behavior of hot pressed lead lanthanum zirconate titanate ceramics, J. Appl. Phys., 1983, 54(6): 3399-3403.

[15] CROSS L E. Relaxor ferroelectrics. Ferroelectrics, 1987, 76(1): 241-267.

[16] SETTER N, CROSS L E. The contribution of structural disorder to diffuse phase transitions in ferroelectrics. J. Mater. Sci., 1980, 15(10): 2478-2482.

[17] TAGANTSEV A K. Vogel-Fulcher relationship for the dielectric permittivity of relaxor ferroelectrics. Phys. Rev. Lett., 1994, 72(7): 1110-1113.

[18] KIMURA T, TOMIOKA Y, KUMAI R, et al. Diffuse phase separation and phase transition in Cr-doped $\mathrm{Nd}_{1 / 2} \mathrm{Ca}_{1 / 2} \mathrm{MnO}_{3}$ : A relaxor ferromagnet. Phys. Rev. Lett., 1999, 83(19): 3940-3943.

[19] OKIMOTO Y, OGIMOTO Y, Matsubara M, et al. Direct observation of photoinduced magnetization in a relaxor ferromagnet. Appl. Phys. Lett., 2002, 80(6): 1031-1033.

[20] KAMZIN A S, BOKOV V A. Mössbauer studies of $\mathrm{PbFe}_{2 / 3} \mathrm{~W}_{1 / 3} \mathrm{O}_{3}$ multiferroics. Phys. Solid State, 2013, 55(6): 1191-1197.

[21] Mitoseriu L, CARnASCiali M M, PIAGgio P, et al. Evidence of the relaxor-paraelectric phase transition in $\mathrm{Pb}\left(\mathrm{Fe}_{2 / 3}\right.$ $\left.\mathrm{W}_{1 / 3}\right) \mathrm{O}_{3}$ ceramics. Appl. Phys. Lett., 2002, 81(26): 5006-5008.

[22] YE Z, TODA K, SATO M, et al. Synthesis, structure and properties of the magnetic relaxor ferroelectric $\mathrm{Pb}\left(\mathrm{Fe}_{2 / 3} \mathrm{~W}_{1 / 3}\right) \mathrm{O}_{3}$ [PFW]. $J$. Korean Phys. Soc., 1998, 32(3): S1028-S1031 .

[23] PAVLENKO A V, TURIKA A V, Reznichenko L A, et al. Dielectric relaxation in the $\mathrm{PbFe}_{1 / 2} \mathrm{Nb}_{1 / 2} \mathrm{O}_{3}$ ceramics. Phys. Solid State, 2011, 53(9): 1872-1875.

[24] CARPENTER M A, SCHIEMER J A, LASCU I, et al. Elastic and magnetoelastic relaxation behaviour of multiferroic (ferromagnetic + ferroelectric + ferroelastic) $\mathrm{Pb}\left(\mathrm{Fe}_{0.5} \mathrm{Nb}_{0.5}\right) \mathrm{O}_{3}$ perovskite. J. Phys.: Condens. Matter, 2015, 27(28): 285901.

[25] BOCHENEK D, GUZDEK P. Ferroelectric and magnetic properties of ferroelectromagnetic $\mathrm{PbFe}_{1 / 2} \mathrm{Nb}_{1 / 2} \mathrm{O}_{3}$ type ceramics. J. Magn. Magn. Mater., 2011, 323(3): 369-374.

[26] LAMPIS N, SCIAU P, LEHMANN A G. Rietveld refinements of the paraelectric and ferroelectric structures of $\mathrm{PbFe}_{0.5} \mathrm{Ta}_{0.5} \mathrm{O}_{3} . J$. Phys.: Condens. Matter, 2000, 12(17): 2367-2378.

[27] DULKIN E, GRUSZKA I, KANIA A, et al. Electric field dependences of Curie and Néel phase transition temperatures in magnetoelectric relaxor multiferroic $\mathrm{Pb}\left(\mathrm{Fe}_{0.5} \mathrm{Ta}_{0.5}\right) \mathrm{O}_{3}$ crystals seen via acoustic emission. Phys. Status Solidi B, 2016, 253(4): 738- 743.

[28] FALQUi A, LAMPIS N, GEDDOLEHMANN A, et al. Lowtemperature magnetic behavior of perovskite compounds $\mathrm{PbFe}_{1 / 2^{-}}$ $\mathrm{Ta}_{1 / 2} \mathrm{O}_{3}$ and $\mathrm{PbFe}_{1 / 2} \mathrm{Nb}_{1 / 2} \mathrm{O}_{3}$. J. Phys. Chem. B, 2005, 109(48): 22967-22970.

[29] KLEEMANN W, SHVARTSMAN V V, BORISOV P, et al. Coexistence of antiferromagnetic and spin cluster glass order in the magnetoelectric relaxor multiferroic $\mathrm{PbFe}_{0.5} \mathrm{Nb}_{0.5} \mathrm{O}_{3}$. Phys. Rev. Lett., 2010, 105(25): 257202.

[30] Chillal S, GVASAliYA S N, ZHELUdEV A, et al. Magnetic short-and long-range order in $\mathrm{PbFe}_{0.5} \mathrm{Ta}_{0.5} \mathrm{O}_{3}$. Phys. Rev. B, 2014, 89(17): 174418.

[31] CHEN L, BOKOV A A, ZHU W M, et al. Magnetoelectric relaxor and reentrant behaviours in multiferroic $\mathrm{Pb}\left(\mathrm{Fe}_{2 / 3} \mathrm{~W}_{1 / 3}\right) \mathrm{O}_{3}$ crystal. Sci. Rep., 2016, 6: 22327.

[32] LEVSTIK A, BOBNAR V, FILIPIČ C, et al. Magnetoelectric relaxor. Appl. Phys. Lett., 2007, 91(1): 012905.

[33] KUMAR A, RIVERA I, KATIYAR R S, et al. Multiferroic $\mathrm{Pb}\left(\mathrm{Fe}_{0.66} \mathrm{~W}_{0.33}\right)_{0.80} \mathrm{Ti}_{0.20} \mathrm{O}_{3}$ thin films: a room-temperature relaxor ferroelectric and weak ferromagnetic. Appl. Phys. Lett., 2008, 92(13): 132913.

[34] CHENG Z X, WANG X L, ALVAREV G, et al. Magnetic glassy behavior in ferroelectric relaxor type solid solutions: magnetoelectric relaxor. J. Appl. Phys., 2009, 105(7): 07D902.

[35] KUMAR A, SHARMA G L, KATIYAR R S, et al. Magnetic control of large room-temperature polarization. J. Phys.: Condens. Matter, 2009, 21(38): 382204.

[36] KUMAR A, KATIYAR R S, SCOTT J F, et al. Fabrication and characterization of the multiferroic birelaxor lead-iron-tungstate/ lead-zirconate-titanate. J. Appl. Phys., 2010, 108(6): 064105.

[37] SANCHEZ D A, KUMAR A, ORTEGA N, et al. Near-room temperature relaxor multiferroic. Appl. Phys. Lett., 2010, 97(20): 202910.

[38] KEMPA M, KAMBA S, SAVINOV M, et al. Bulk dielectric and magnetic properties of PFW? PZT ceramics: absence of magnetically switched-off polarization. J. Phys.: Condens. Matter, 2010, 22(44): 445902.

[39] CATALAN G, SCOTT J F. Physics and application of bismuth ferrite. Adv. Mater., 2009, 21: 2463-2485.

[40] PALEWICZ A, PRZENIOSLO R, SOSNOWSKA I, et al. Atomic displacements in $\mathrm{BiFeO}_{3}$ as a function of temperature: neutron diffraction study. Acta Crystallogr. B, 2007, 63(4): 537-544.

[41] NEATON J, EDERER C, WAGHMARE U, et al. First-principles 
study of spontaneous polarization in multiferroic $\mathrm{BiFeO}_{3}$. Phys. Rev. B, 2005, 71(1): 014113.

[42] LEBEUGLE D, COLSON D, FORGET A, et al. Room-temperature coexistence of large electric polarization and magnetic order in $\mathrm{BiFeO}_{3}$ single crystals. Phys. Rev. B, 2007, 76(2): 024116.

[43] ZHANG ST, LU M H, WU D, et al. Larger polarization and weak ferromagnetism in quenched $\mathrm{BiFeO}_{3}$ ceramics with a distorted rhombohedral crystal structure. Appl. Phys. Lett, 2005, 87(26): 262907.

[44] ARNOLD D C, KNIGHT K S, CATALAN G, et al. The $\beta$-to- $\gamma$ transition in $\mathrm{BiFeO}_{3}$ : a powder neutron diffraction study. Adv. Fun. Mater., 2010, 20(13): 2116-2123.

[45] MIAO J, ZHANG X, ZHAN Q, et al. Bi-relaxation behaviors in epitaxial multiferroic double-perovskite $\mathrm{BiFe}_{0.5} \mathrm{Mn}_{0.5} \mathrm{O}_{3} / \mathrm{CaRuO}_{3}$ heterostructures. Appl. Phys. Lett., 2011, 99(6): 062905.

[46] WEI Y X, WANG X T, ZHU J T, et al. Dielectric, ferroelectric and piezoelectric properties of $\mathrm{BiFeO}_{3}-\mathrm{BaTiO}_{3}$ ceramics. J. Am. Ceram. Soc., 2013, 96(10): 3163-3168.

[47] WEI Y X, WANG X T, JIA J J, et al. Multiferroic and piezoelectric properties of $0.65 \mathrm{BiFeO}_{3}-0.35 \mathrm{BaTiO}_{3}$ ceramic with pseudo-cubic symmetry. Ceram. Int., 2013, 38(4): 3499-3502.

[48] VERMA K C, KOTNALA R K. Multiferroic magnetoelectric coupling and relaxor ferroelectric behavior in $0.7 \mathrm{BiFeO}_{3}-0.3 \mathrm{BaTiO}_{3}$ nanocrystals. Solid State Commun., 2011, 151(13): 920-923.

[49] OZAKI T, KITAGAWA S, NISHIHARA S, et al. Ferroelectric properties and nano-scaled domain structures in $(1-x) \mathrm{BiFeO}_{3}$ $x \mathrm{BaTiO}_{3}(0.33<x<0.50)$. Ferroelectrics, 2009, 385: 155-161.

[50] SODA M, MATSUURA M, WAKABAYASHI W, et al. Superparamagnetism induced by polar nanoregions in relaxor ferroelectric (1- $x) \mathrm{BiFeO}_{3}-x \mathrm{BaTiO}_{3}$. J. Phys. Soc. Jpn., 2011, 80(4): 043705.

[51] BHATTACHAEJEE S, TRIPATHI S, PANDEY D. Morphotropic phase boundary in $(1-x) \mathrm{BiFeO}_{3}-\chi \mathrm{PbTiO}_{3}$ : phase coexistence region and unusually large tetragonality. Appl. Phys. Lett., 2007, 91(4): 042903.

[52] ZHU W M, GUO H Y, YE Z G. Structural and magnetic characterization of multiferroic $\left(\mathrm{BiFeO}_{3}\right)_{(1-x)}\left(\mathrm{PbTiO}_{3}\right)_{x}$ solid solutions. Phys. Rev. B, 2008, 78(1): 014401.

[53] AMORÍN H, CORREAS C, RAMOS P, et al. Very high remnant polarization and phase-change electromechanical response of Bi$\mathrm{FeO}_{3}-\mathrm{PbTiO}_{3}$ at the multiferroic morphotropic phase boundary. Appl. Phys. Lett., 2012, 101(17): 172908.

[54] SINGH A, GUPTA A, CHATTERJEE R. Enhanced magnetoelectric coefficient $(\alpha)$ in the modified $\mathrm{BiFeO}_{3}-\mathrm{PbTiO}_{3}$ system with large La substitution. Appl. Phys. Lett., 2008, 93(2): 022902.

[55] MATSUO H, NOGUCHI Y, MIYAYAMA M, et al. Structural and piezoelectric properties of high-density $\left(\mathrm{Bi}_{0.5} \mathrm{~K}_{0.5}\right) \mathrm{TiO}_{3}-\mathrm{BiFeO}_{3}$ ceramics. J. Appl. Phys., 2010, 108(10): 104103.

[56] BENNETT J, BELL A J, STEVENSON T, et al. Multiferroic properties of $\mathrm{BiFeO}_{3}-\left(\mathrm{K}_{0.5} \mathrm{Bi}_{0.5}\right) \mathrm{TiO}_{3}$ ceramics. Mater. Lett., 2013, 94(3): $172-175$.

[57] MOROZOV M I, EINARSRUD M, GRANDE T. Polarization and strain response in $\mathrm{Bi}_{0.5} \mathrm{~K}_{0.5} \mathrm{TiO}_{3}-\mathrm{BiFeO}_{3}$ ceramics. Appl. Phys. Lett., 2012, 101(25): 252904.

[58] DORCET V, MARCHET P, TROLLIARD G, et al. Structural and dielectric studies of the $\mathrm{Na}_{0.5} \mathrm{Bi}_{0.5} \mathrm{TiO}_{3}-\mathrm{BiFeO}_{3}$ system. J. Eur. Ceram. Soc., 2007, 27(13/14/15): 4371-4374.

[59] RAMANA E V, SURYANARAYANA S V, SANKARAM T B, et al. Synthesis and magnetoelectric studies on $\mathrm{Na}_{0.5} \mathrm{Bi}_{0.5} \mathrm{TiO}_{3}-\mathrm{BiFeO}_{3}$ solid solution ceramics. Solid State Sci., 2010, 12(5): 956-962.

[60] HENRICHS L F, CESPEDES O, BENNETT J, et al. Multiferroic clusters: a new perspective for relaxor-type room-temperature multiferroics. Adv. Fun. Mater., 2016, 26(13): 2111-2121.

[61] SHVARTSMAN V V, BEDANTA S, BORISOV P, et al. $\mathrm{SrMnTiO}_{3}$ : a magnetoelectric multiglass. Phys. Rev. Lett., 2008, 101(16): 165704 .

[62] MULLER K A, BURKARD H. $\mathrm{SrTiO}_{3}$ : an intrinsic quantum paraelectric below 4 K. Phys. Rev. B, 1979, 19(7): 3593-3602.

[63] BEDNORZ J G, MULLER K A. $\mathrm{Sr}_{1-x} \mathrm{Ca}_{x} \mathrm{TiO}_{3}$ : an XY quantum ferroelectric with transitions to randomness. Phys. Rev. Lett., 1984, 52(25): 2289-2292.

[64] TKACH A, VILARINHO P M, KHOLKIN A L. Polar behavior in Mndoped $\mathrm{SrTiO}_{3}$ ceramics. Appl. Phys. Lett., 2005, 86(17): 172902.

[65] TKACH A, VILARINHO P M, KHOLKIN A L, et al. Structuremicrostructure-dielectric tunability relationship in Mn-doped strontium titanate ceramics. Acta Mater., 2005, 53(19): 5061-5069.

[66] LI J B, HUANG Y P, RAO G H, et al. Ferroelectric transition of Aurivillius compounds $\mathrm{Bi}_{5} \mathrm{Ti}_{3} \mathrm{FeO}_{15}$ and $\mathrm{Bi}_{6} \mathrm{Ti}_{3} \mathrm{Fe}_{2} \mathrm{O}_{18}$. Appl. Phys. Lett., 2010, 96(22): 222903.

[67] BAI W, YIN W B, YANG J, et al. Cryogenic temperature relaxor-like dielectric responses and magnetodielectric coupling in Aurivillius $\mathrm{Bi}_{5} \mathrm{Ti}_{3} \mathrm{FeO}_{15}$ multiferroic thin films. J. Appl. Phys., 2014, 116(8): 084103.

[68] HERVOCHES C H, SNEDDEN A, RIGGS R, et al. Structural behavior of the four-layer Aurivillius-phase ferroelectrics $\mathrm{SrBi}_{4} \mathrm{Ti}_{4} \mathrm{O}_{15}$ and $\mathrm{Bi}_{5} \mathrm{Ti}_{3} \mathrm{FeO}_{15}$. J. Solid State Chem., 2002, 164(2): 280-291.

[69] ZHAO H Y, KIMURA H, CHENG Z X, et al. Large magnetoelectric coupling in magnetically short-range ordered $\mathrm{Bi}_{5} \mathrm{Ti}_{3} \mathrm{FeO}_{15}$ film. Sci. Rep., 2014, 4: 5255.

[70] HEMBERGER J, LUNKENHEIMER P, FICHTL R, et al. Relaxor ferroelectricity and colossal magnetocapacitive coupling in ferromagnetic $\mathrm{CdCr}_{2} \mathrm{~S}_{4}$. Nature, 2005, 434(7031): 364-367.

[71] CATALAN G, SCOTT J F. Magnetoelectrics: is $\mathrm{CdCr}_{2} \mathrm{~S}_{4}$ a multiferroic relaxor? Nature, 2007, 448(7156): E4-E5.

[72] WALZ F. The Verwey transition-a topical review. J. Phys.: Condens. Matter, 2002, 14(12): R285-R340.

[73] KATO K, IIDA S, YANAI K, et al. Ferrimagnetic ferroelectricity of $\mathrm{Fe}_{3} \mathrm{O}_{4}$. J. Magn. Magn. Mater., 1983, 31-34(6): 783-784.

[74] ZIESE M, ESQUINAZI P D, PANTEL D, et al. Magnetite $\left(\mathrm{Fe}_{3} \mathrm{O}_{4}\right)$ : a new variant of relaxor multiferroic? J. Phys.: Condens. Matter, 2012, 24(8): 086007.

[75] FIER I, WALMSLEY L, SOUZA J A. Relaxor behavior in multiferroic $\mathrm{BiMn}_{2} \mathrm{O}_{5}$ ceramics. J. Appl. Phys., 2011, 110(8): 084101. 